archives-ouvertes

\title{
Conception d'un entraînement électrique à aimants permanents pour une chaîne de traction de véhicule hybride électrique
}

\author{
Zhenwei Wu, Daniel Depernet, Christophe Espanet
}

\section{To cite this version:}

Zhenwei Wu, Daniel Depernet, Christophe Espanet. Conception d'un entraînement électrique à aimants permanents pour une chaîne de traction de véhicule hybride électrique. European Journal of Electrical Engineering (EJEE), 2011, 14 (2-3), pp.215 - 236. hal-02300090

\section{HAL Id: hal-02300090 \\ https://hal.archives-ouvertes.fr/hal-02300090}

Submitted on 29 Sep 2019

HAL is a multi-disciplinary open access archive for the deposit and dissemination of scientific research documents, whether they are published or not. The documents may come from teaching and research institutions in France or abroad, or from public or private research centers.
L'archive ouverte pluridisciplinaire HAL, est destinée au dépôt et à la diffusion de documents scientifiques de niveau recherche, publiés ou non, émanant des établissements d'enseignement et de recherche français ou étrangers, des laboratoires publics ou privés. 


\title{
Conception d'un entraînement électrique à aimants permanents pour une chaîne de traction de véhicule hybride électrique
}

\author{
Zhenwei Wu $^{1,3}$ - Daniel Depernet ${ }^{2,3}$ - Christophe Espanet ${ }^{1,3}$ \\ ${ }^{1}$ Université de Franche-Comté, FEMTO-ST, Belfort, France \\ ${ }^{2}$ Université de Technologie de Belfort-Montbéliard, FEMTO-ST, Belfort, France \\ ${ }^{3}$ MEGEVH Projet National Français en Véhicule Hybride Électrique \\ zwu@univ-fcomte.fr
}

RÉSUMÉ. Dans cet article, les auteurs décrivent une méthode de modélisation et de dimensionnement d'un entrainement électrique à aimants permanents utilisé dans une chaîne de traction pour Véhicule Hybride Electrique (VHE) de type poids lourds et basée sur une architecture série parallèle. La résolution analytique du dimensionnement géométrique de la Machine Synchrone à Aimants Permanents Surfaciques (MSAPS), du calcul des grandeurs électriques et des pertes dans la machine et l'onduleur permet l'évaluation paramétrée des caractéristiques de la chaîne de traction. Les performances sont ensuite validées à l'aide d'une Analyse par Eléments Finis (AEF) de la MSAPS. Les pertes totales et le rendement global de l'ensemble machine onduleur sont évalués pour différents points de fonctionnements, en fonction de plusieurs paramètres tels que la tension de bus ou la fréquence de commutation de l'onduleur. La méthodologie présentée dans ce papier est générale et peut être une première étape vers le dimensionnement optimal du train de puissance complet, incluant l'entrainement électrique mais aussi le réducteur planétaire, les éléments de stockage de l'énergie embarquée et éventuellement le moteur thermique.

ABSTRACT. In this paper, the authors describe a method of modeling and design of a permanent magnet drive used in a power train for Hybrid Electric Heavy Vehicle, based on a Series-Parallel architecture. In this paper, a Surface-Mounted Permanent Magnet Synchronous Machine is considered. The geometric design, the electric parameters and the losses in the motor and the inverter are analytically calculated in order to evaluate the characteristics of the powertrain. The motor performances are validated with Finite Element Analysis. The total losses and the efficiency of the motor and the inverter are evaluated for several working points. This methodology is general and can be a first step of a complete power train optimal design, including the electrical drive, the gear, the energy storage units and possibly the internal combustion engine.

MOTS-CLÉS: Modélisation analytique (MA), Véhicule Hybride Electrique Série-Parallèle, MSAPS, Onduleur de Tension, Pertes, Rendement.

KEYWORDS: Analytical modelling, Hybrid Electric Heavy Vehicle, SMPMSM, Inverter, Losses, efficiency

DOI:10.3166/Geo.19.11-38

Revue. Volume $\mathrm{X}-\mathrm{n}^{\circ} \mathrm{x} /$ année, pages 1 à $\mathrm{X}$ 
2 Revue. Volume $\mathrm{X}-\mathrm{n}^{\circ} \mathrm{x} /$ année 


\section{Introduction}

De nombreuses solutions ont déjà été proposées pour le développement de systèmes de propulsion de Véhicules Hybrides Electriques (VHE). Généralement, les motorisations des VHE sont classées en 4 types (Chan, 2002, Mester, 2007, Chan, 2010): la motorisation hybride série, la motorisation hybride parallèle, la motorisation hybride série-parallèle, la motorisation hybride complexe. Toutefois, nous pouvons constater que les solutions ont principalement été développées pour des véhicules légers (voitures urbaines ou de tourisme). Dans ce contexte, les travaux de recherche dans lesquels s'insère le travail présenté dans ce papier portent sur les VHE lourds et ont pour objectif de développer une méthodologie de modélisation analytique et de dimensionnement optimal adaptée aux chaînes de traction hybrides (Taylor et al., 2001). Comme la masse du VHE étudié est de plus de 20 tonnes, le couple et la puissance nécessaires sont élevés afin de subvenir aux régimes de fonctionnement tels que le démarrage ou le mode boost (fortes accélérations). Parmi les architectures existantes, notre choix se porte sur la structure Série-Parallèle (Figure 1) qui présente une plus grande souplesse de fonctionnement que ses concurrentes. En effet, il existe dans ce cas un degré de liberté permettant de régler relativement librement la répartition entre les flux d'énergie électriques et thermiques et, malgré le nombre important de composants installés, cette structure présente un bon compromis technico-économique entre la puissance totale installée et la puissance maximale réellement disponible pour la propulsion.

Le fonctionnement d'une propulsion hybride Série-Parallèle est essentiellement constitué de 4 phases qui sont illustrées dans les Figures 2 à 5. L'interconnexion du moteur thermique, des machines électriques et des roues, et donc la composition des puissances électriques et mécaniques, est assurée par un train épicycloïdal double dit train planétaire de Ravigneaux. Pour des raisons de simplicité de réalisation, les machines électriques 1 et 2 sont identiques et, quoique réversibles, elles jouent respectivement le rôle principal de génératrice et de moteur.

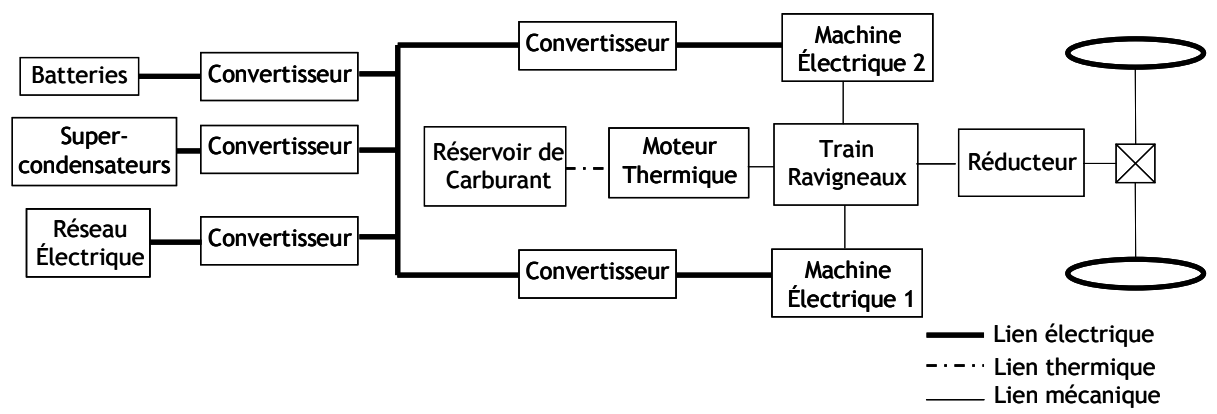

Figure 1. Structure Série-Parallèle du VHE 
- Fonctionnement tout électrique (Figure 2) : Le moteur thermique est arrêté et le réseau électrique alimente la machine électrique 2 qui fonctionne en moteur et entraîne les roues.

- Fonctionnement en mode thermique (Figure 3) : La force motrice du moteur thermique se répartit en deux branches grâce au train épicycloïdal : une partie de la puissance du moteur thermique est transmise directement aux roues et l'autre à la machine électrique 2 par l'intermédiaire de la machine électrique 1 (fonctionnant en génératrice) et du réseau électrique. Le pilotage des machines électriques permet d'assurer l'équilibrage des couples agissant sur le train épicycloïdal double.

- Fonctionnement en forte accélération, appelé également Boost (Figure 4) : Ce régime de fonctionnement consiste à additionner la puissance développée par le moteur thermique à celle provenant du réseau électrique. L'énergie provenant des sources électriques est appliquée aux roues par l'intermédiaire de la machine électrique 2 fonctionnant en moteur. L'équilibrage des couples agissant sur le train épicycloïdal est là encore assuré par le pilotage des deux machines électriques. Le mode recharge des batteries est comparable au mode Boost (Figure 4): l'énergie provenant du moteur thermique se partage entre les roues et le réseau électrique afin d'assurer la recharge des batteries par l'intermédiaire de la génératrice électrique.

- Fonctionnement en mode récupération (Figure 5) : C'est la phase de récupération d'énergie. Les roues transmettent l'énergie cinétique du véhicule en entraînant la machine électrique 2 fonctionnant en génératrice et en ralentissant ainsi le véhicule. L'énergie électrique transmise au réseau électrique est récupérée ou dissipée.

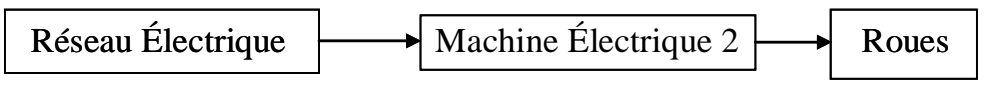

Figure 2. Mode tout électrique

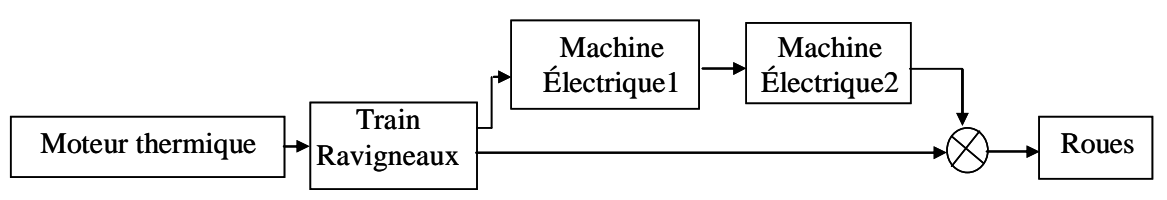

Figure 3. Mode thermique

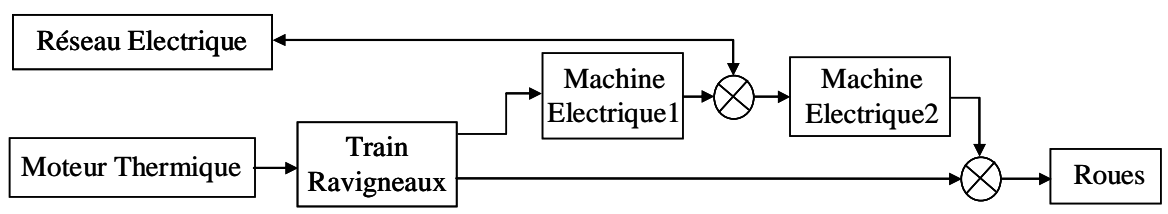

Figure 4. Mode Boost et recharge des batteries 


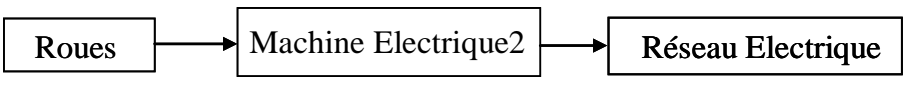

Figure 5. Mode récupération

Dans cet article, les composants électriques de la chaîne de traction décrite cidessus sont modélisés afin de permettre son dimensionnement. Les modèles sont analytiques afin d'aboutir à une résolution rapide des équations dans un premier temps et afin de rendre aisée leur intégration dans une optimisation de la chaîne de traction (par algorithme mathématique de minimisation de critères) dans un deuxième temps. Cet article décrit les méthodes de modélisation analytique (MA) des quatre modèles suivants :

- $\quad$ le modèle de pré-dimensionnement des machines électriques, permettant le dimensionnement de la motorisation électrique à aimants permanents ;

- $\quad$ le modèle électromécanique, permettant le calcul des grandeurs électriques caractérisant la motorisation électrique et son onduleur d'alimentation ;

- $\quad$ le modèle de pertes dans la motorisation électrique ;

- le modèle de pertes dans l'onduleur d'alimentation des machines électriques.

Ces modèles s'imbriquent pour permettre un dimensionnement global de la propulsion électrique en obtenant non seulement les paramètres géométriques de dimensionnement (et donc l'encombrement et la masse de la machine), mais également les performances énergétiques de l'ensemble constitué par les moteurs électriques et leur électronique d'alimentation (Figure 6).

Le dimensionnement des machines électriques est réalisé en considérant 3 points de fonctionnements caractérisant à la fois les limites dimensionnantes et les modes de fonctionnement les plus représentatifs du profil de mission du véhicule :

- $\quad$ Point 1 : Fonctionnement transitoire (moins de 30s) à la moitié de la vitesse nominale et au double du couple nominal. Ce point correspond au fonctionnement du moteur en mode boost. Pendant cette phase, le véhicule doit pouvoir accélérer de 0 à $30 \mathrm{~km} / \mathrm{h}$ en moins de $30 \mathrm{~s}$. La puissance correspondante vaut $70 \mathrm{~kW}$.

- $\quad$ Point 2 : Fonctionnement à vitesse et couple nominaux.

- $\quad$ Point 3 : Fonctionnement à vitesse maximale. Le couple est défini par la limitation de puissance à $70 \mathrm{~kW}$.

Nous donnons Figure 7 la caractéristique typique du couple électromagnétique en fonction de la vitesse de rotation du moteur sur laquelle nous avons également représenté les 3 points de fonctionnements. Dans cet exemple, la vitesse nominale est de $668 \mathrm{tr} / \mathrm{min}$ et le couple nominal de $1000 \mathrm{~N} \cdot \mathrm{m}$. 


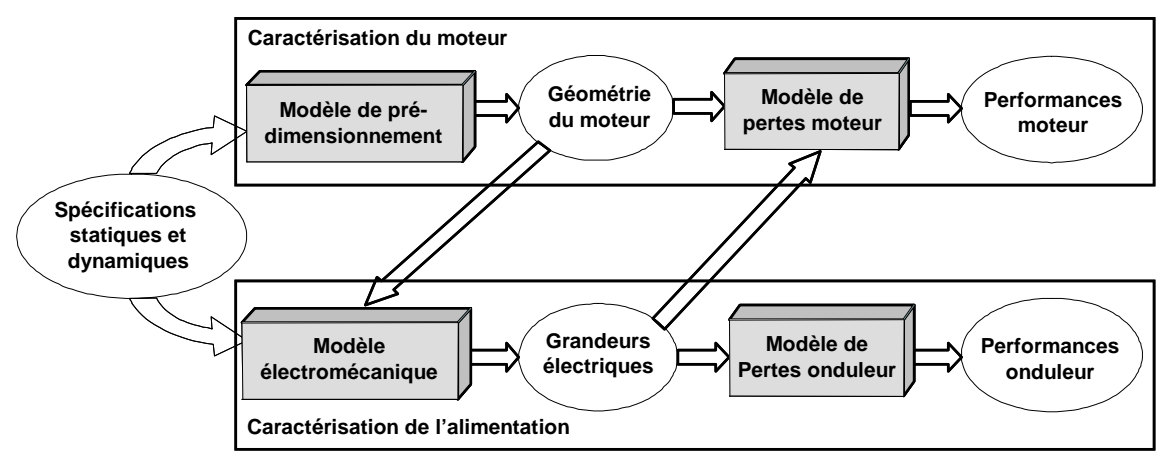

Figure 6. Principe de dimensionnement par modélisation analytique

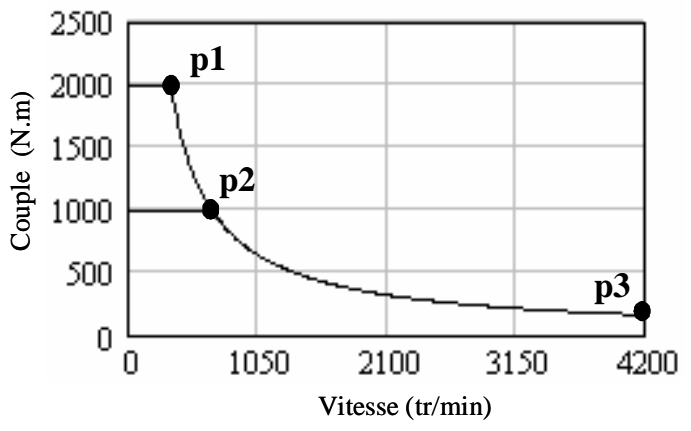

Figure 7. Caractéristique du couple en fonction de la vitesse de rotation

\section{Modèle de pré-dimensionnement analytique de la MSAPS}

Les auteurs ont retenu la machine à aimants montés en surface pour la mise en place de la méthodologie de modélisation et de dimensionnement. Même si ce choix n'est pas optimal du point de vue du dimensionnement du convertisseur (la réduction du flux à vitesse élevée nécessite un fort courant réactif du fait de la faiblesse de l'inductance cyclique), l'objectif est de proposer la méthodologie de modélisation et de dimensionnement et de l'appliquer à un premier choix de machine électrique. De futurs développements concerneront des machines à aimants enterrés et à concentration de flux.

Quoi qu'il en soit, de nombreux auteurs ont étudié le dimensionnement analytique de la machine à aimants permanents montés en surface (par exemple Lechevallier et al., 2007, Slemon et al., 1992, Regnier, 2003, Zhu et al., 2008, Slemon, 1994, Espanet, 1999). Une modélisation analytique de la machine basée sur les approches détaillées dans (Slemon et al., 1992) a ainsi été développée afin de 
réaliser le pré-dimensionnement du moteur à partir des données du cahier des charges (Brisset, 2001, Gasc, 2004).

\subsection{Principe du modèle analytique}

Nous développons ici brièvement les équations analytiques qui permettent de calculer les dimensions géométriques de la machine en fonction des contraintes nominales et maximales de couple, puissance, tension, échauffement ou encombrement.

En appliquant le théorème d'Ampère, nous exprimons l'induction d'entrefer créée par les aimants :

$B_{g}=B_{r} \frac{e_{m g} / \mu_{r}}{K_{c} g+e_{m g} / \mu_{r}}$

où $\mu_{r}$ est la perméabilité magnétique relative et $\mathrm{B}_{\mathrm{r}}=1.2 \mathrm{~T}$ la valeur d'induction rémanente des aimants. Le paramètre $\mathrm{e}_{\mathrm{mg}}$ est le rapport adimensionnel de l'épaisseur de l'aimant sur l'entrefer corrigé. L'entrefer corrigé est classiquement obtenu en tenant compte des effets de denture statorique par l'intermédiaire du coefficient de Carter.

Le couple électromagnétique peut s'exprimer comme suit :

$$
C_{e m}=\pi \cdot r_{s}^{2} \cdot l_{r} \cdot B_{g} \cdot d_{s} \cdot J_{s} \cdot K_{r} \cdot K_{b}
$$

où $\mathrm{d}_{\mathrm{s}}$ représente la profondeur d'encoche, $\mathrm{l}_{\mathrm{r}}$ la longueur active du moteur, $\mathrm{J}_{\mathrm{s}}$ la valeur efficace de la densité de courant surfacique dans les encoches, $\mathrm{K}_{\mathrm{r}}$ le coefficient de remplissage de cuivre dans les encoches et $\mathrm{K}_{\mathrm{b}}$ est le coefficient de bobinage. Ainsi, le rayon d'alésage $\mathrm{r}_{\mathrm{s}}$ peut s'exprimer à partir de l'expression du couple :

$r_{s}=\left(\frac{C_{e m} \cdot R_{r l}}{J_{s} \cdot K_{r} \cdot B_{g} \cdot R_{d r} \cdot \pi}\right)^{\frac{1}{4}}$

Dans cette expression, $\mathrm{R}_{\mathrm{dr}}$ représente le rapport entre la profondeur d'encoche et le rayon d'alésage du moteur, $\mathrm{R}_{\mathrm{rl}}$ le rapport entre le rayon d'alésage du moteur et la longueur active du moteur. Nous pouvons ensuite déterminer toutes les autres caractéristiques géométriques, mécaniques, électriques et magnétiques (Slemon, 1994).

\subsection{Caractéristiques du moteur dimensionné}

La figure 8 représente les dimensions géométriques de la machine utilisée dans la MA de pré-dimensionnement. Ainsi, en exploitant le modèle décrit ci-dessus 
appliqué au cahier des charges décrit dans l'introduction, on obtient la machine électrique dont les principaux paramètres géométriques sont donnés dans le tableau 1. Cette solution est obtenue à partir d'une étude paramétrique visant à minimiser la masse de la motorisation tout en respectant les contraintes d'intégration au sein du système de transmission du véhicule. Ces contraintes d'intégration mécanique sont traduites numériquement par des valeurs limites des paramètres $R_{\mathrm{dr}}$ et $\mathrm{R}_{\mathrm{rl}}$ qui sont dans un intervalle de $(0,10)$ qui correspondent à des limites de faisabilité.

Compte tenu des limites de la MA pour caractériser finement le comportement électromagnétique, notamment vis-à-vis de la saturation des différents matériaux magnétiques, les résultats obtenus sont vérifiés à l'aide d'une $\mathrm{AEF}$ (Cecco, 2005). Le tableau 2 donne une comparaison des propriétés de la machine obtenues par MA et AEF dans le cas de l'exemple décrit précédemment. L'AEF permet d'effectuer une validation des solutions obtenues par la MA assurant le respect des performances et des contraintes. On peut noter une bonne concordance générale des principaux paramètres donnés par la MA et l'AEF, les disparités étant principalement dues à la meilleure représentation des phénomènes de saturation par l'AEF. On peut noter toutefois que l'obtention d'un couple très proche de la valeur attendue au point nominal traduit un très faible comportement saturé de la machine, et que ce phénomène reste raisonnable dans le cas d'un fonctionnement en mode boost. Cette propriété permet de valider l'efficacité de la machine électrique et en particulier de vérifier la capacité du moteur à produire réellement le couple désiré, ans adjonction excessive de courant supplémentaire, source de pertes et pouvant affecter le rendement.

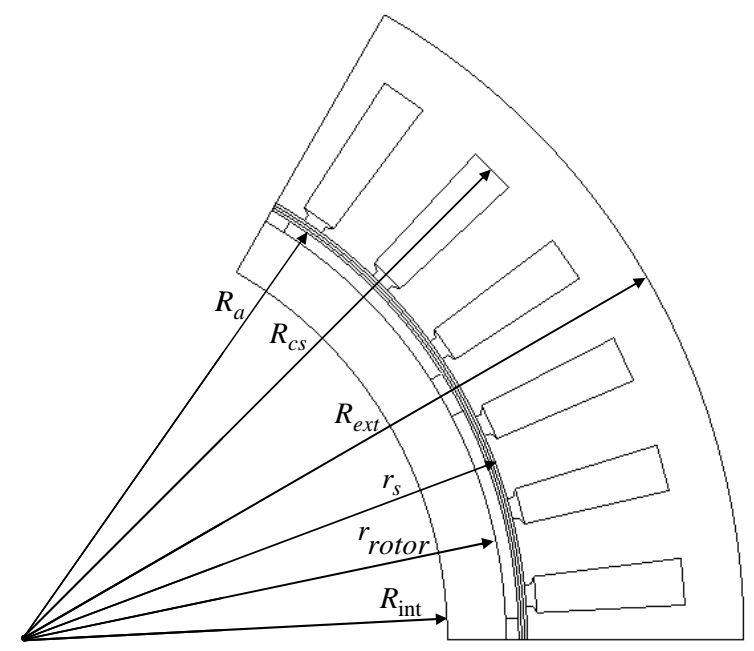

Figure 8. Structure géométrique de la MSAPS 
Tableau 1. Paramètres géométriques d'une MSAPS dimensionnée pour le train de puissance d'un véhicule lourd de type hybride électrique série-parallèle

\begin{tabular}{ccc}
\hline Paramètre & Symbole & Valeur \\
\hline Rayon intérieur du rotor & $R_{\mathrm{int}}$ & $99.9(\mathrm{~mm})$ \\
Rayon extérieur du rotor & $r_{\text {rotor }}$ & $113.7(\mathrm{~mm})$ \\
Rayon extérieur d'aimants & $R_{a}$ & $116.7(\mathrm{~mm})$ \\
Épaisseur d'aimants & $e_{m}$ & $3(\mathrm{~mm})$ \\
Entrefer & $g$ & $2(\mathrm{~mm})$ \\
Rayon intérieur du stator & $r_{s}$ & $118.7(\mathrm{~mm})$ \\
Rayon extérieur du stator & $R_{c s}$ & $156.2(\mathrm{~mm})$ \\
Payon intérieur de la culasse statorique & $R_{e x t}$ & $170(\mathrm{~mm})$ \\
Londeur des dents statoriques & $d_{s}$ & $37.4(\mathrm{~mm})$ \\
Rapport du profondeur d'encoche au rayon & $R_{d r}$ & $485.8(\mathrm{~mm})$ \\
d'alésage & & 0.315 \\
Rapport du rayon d'alésage à la longueur & $R_{r l}$ & 0.244 \\
active de la machine & &
\end{tabular}

Tableau 2. Comparaison des Performances Obtenues par MA et AEF

\begin{tabular}{|c|c|c|c|}
\hline & MA & $\mathrm{AEF}$ & Erreur $(\%)$ \\
\hline $\begin{array}{l}\text { Fondamendal de la force } \\
\text { éctromotrice }(\mathrm{V})\end{array}$ & 229.9 & 232.9 & 1.3 \\
\hline $\begin{array}{c}\text { Couple électromagnétique dans les } \\
\text { conditions nominales d'alimentation (N.m) }\end{array}$ & 1000 & 993 & 0.7 \\
\hline $\begin{array}{c}\text { Couple électromagnétique dans les } \\
\text { conditions d'alimentation en boost (N.m) }\end{array}$ & 2000 & 1886 & 5.7 \\
\hline
\end{tabular}

\section{Modélisation électromécanique}

La modélisation analytique du comportement électromécanique de la machine permet d'étudier des grandeurs électriques de l'ensemble convertisseur - machine. Elle consiste à exprimer, à partir d'un schéma monophasé équivalent (en régime sinusoïdal permanent), les lois de commande en couple et vitesse de la machine 
électrique, les lois de commande en courant pour créer le couple désiré à partir des équations électriques en régime permanent sinusoïdal équilibré, et les contraintes liées à l'utilisation d'un onduleur de tension à 2 niveaux (Capitaneanu, 2002). Les caractéristiques du moteur issues de la MA, les points de fonctionnement nominaux et les caractéristiques des composants de l'onduleur de tension constituent les paramètres d'entrée du modèle électromécanique. Les lois de couple et de puissance en fonction de la vitesse sont représentées Figure 9.

(a)

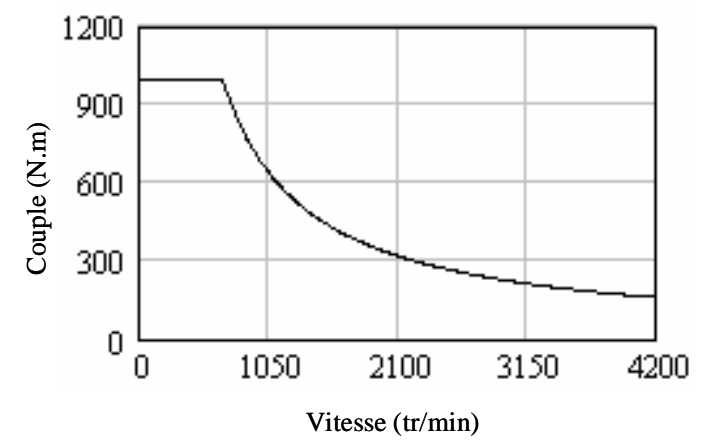

(b)

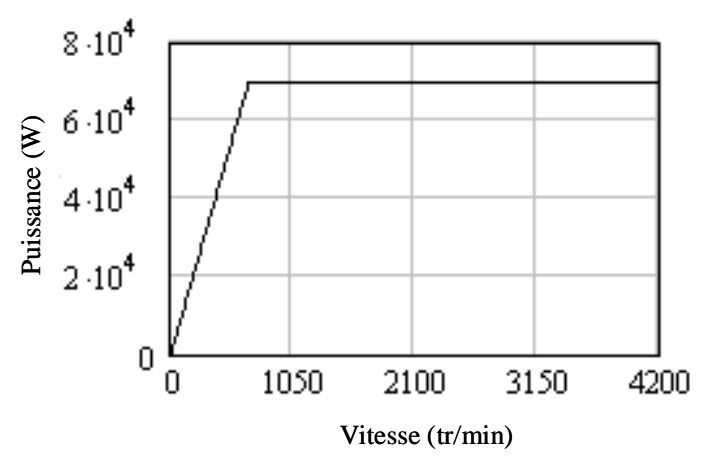

Figure 9. Lois de (a) couple et (b) de puissance du moteur électrique

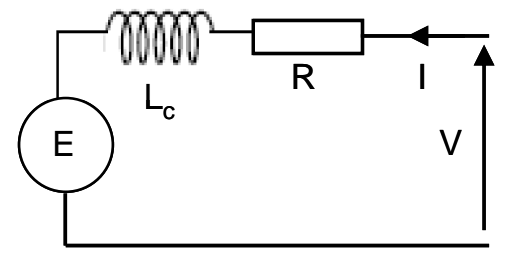

avec

$\mathrm{R}$ : résistance d'une phase

$\mathrm{L}_{\mathrm{c}}$ : inductance cyclique d'une phase

$\mathrm{V}$ : tension simple d'alimentation

E : f.é.m. d'une phase

Figure 10. Modèle d'une phase du moteur électrique 
Le modèle de la MSAPS représenté Figure 10 permet d'exprimer les équations électriques [4] liant courants, tensions et couple électromagnétique.

$\left\{\begin{array}{l}V=E+\left(R+j L_{c} \cdot p \cdot \Omega\right) \cdot I=V_{d}+j V_{q} \\ C_{e m}=3 \cdot p \cdot \Phi_{\text {eff }} \cdot I_{\text {eff }} \cdot \cos (\psi)\end{array}\right.$

avec $I=I_{d}+j I_{q}$

où $p$ représente le nombre de paires de pôle, $\Omega$ la vitesse angulaire, $\mathrm{C}_{\mathrm{em}}$ le couple électromagnétique, $\Phi_{\text {eff }}$ le flux efficace, $\mathrm{I}_{\text {eff }}$ le courant efficace dans une phase et $\psi$ le déphasage entre I et $\mathrm{E}$.

La composante de courant $I_{q}$ en phase avec la f.é.m. permet de créer le couple maximal. Ainsi, la loi de commande de la machine utilisée consiste, lorsque c'est possible, à imposer un courant en phase avec la f.é.m. afin de minimiser son amplitude $\left(I_{d}=0\right)$. Ceci permet de minimiser les pertes dans la machine et dans l'onduleur. Cependant, l'alimentation par onduleur de tension de la MSAPS induit une contrainte de limitation en amplitude de la tension appliquée à la machine. A vitesse élevée, il est alors nécessaire de déphaser le courant par rapport à la f.é.m. pour conserver le couple requis compte tenu de la tension que peut délivrer l'onduleur. La composante $I_{d}$ est alors calculée afin d'assurer une compensation partielle du flux créé par les aimants de la machine et assurant le pilotage en couple et en vitesse par l'onduleur malgré sa limitation en tension. Le courant est dans ce cas encore maintenu à sa valeur minimale requise pour minimiser les pertes dans le moteur et l'onduleur. Les évolutions de l'amplitude de la tension en sortie d'onduleur et du courant dans la machine en fonction de la vitesse sont représentées sur les Figure 11. (a) et (b) dans le cas d'une tension continue de 540V en entrée de l'onduleur. 

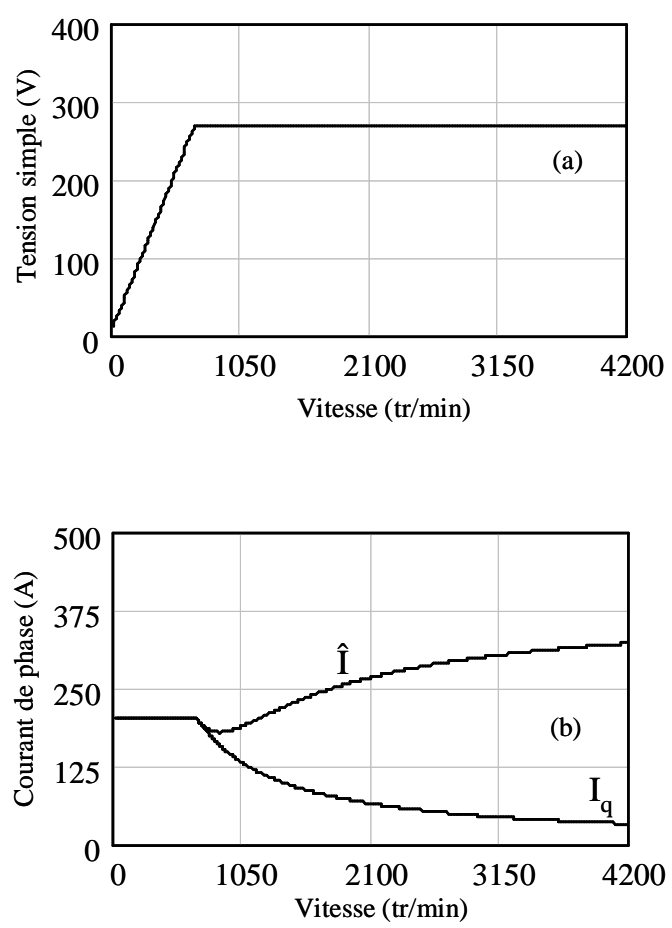

Figure 11. Loi de contrôle de la machine. (a). Amplitude de la tension simple.

(b). Amplitude du courant de phase et composante active

Le modèle électromécanique permet ainsi d'évaluer rapidement le comportement électrique de la machine à partir de ses principaux paramètres et des caractéristiques de l'onduleur. Il est par exemple utile de quantifier l'impact de la limitation de tension sur l'amplitude du courant permettant le défluxage de la machine. Les critères de choix peuvent donc plus facilement être définis et pris en compte lors de la conception de l'ensemble convertisseur et machine afin de minimiser les pertes globales dues aux courants.

Par ailleurs, ce modèle permettrait une comparaison des pertes induites par le défluxage dans le cas de solutions d'intégration concurrentes des aimants permanents telles que les structures à aimants enterrés plus facilement défluxables. Il faudrait toutefois modifier le schéma monophasé équivalent en faisant intervenir les inductances dans les axes directs et transverses qui ne sont généralement pas identiques dans le cas de machines à aimants enterrés. 


\section{Modélisation des pertes dans les deux machines électriques}

Par hypothèse, les pertes magnétiques dans les aimants et dans le rotor dues aux courants de Foucault sont négligées. Dans ces conditions, les pertes dans le moteur électrique sont composées par les pertes fer dans les dents statoriques, les pertes fer dans la culasse statorique et les pertes cuivre dans le bobinage du stator (Espanet, 1999). L'induction magnétique dans les différentes parties du moteur est un élément important pour calculer les pertes dans le moteur. Nous avons considéré que l'induction magnétique est parfaitement sinusoïdale dans toutes les parties du moteur.

Le défluxage du moteur par le courant au stator est pris en compte dans la MA du calcul des pertes fer dans la culasse statorique. Lors d'un fonctionnement du moteur en charge, le courant du bobinage $I_{d}$ crée un champ magnétique qui influe le champ total dans la machine. Ainsi, l'induction résultante $\mathrm{B}_{\text {statc }}$ dans le stator lors d'un fonctionnement en charge peut s'exprimer comme suit :

$B_{\text {statc }}=B_{\text {statv }}\left(1-\frac{L_{c} \cdot I_{d}}{\sqrt{2} \cdot \Phi_{\text {eff }}}\right)$

où $\mathrm{B}_{\text {statv }}$ représente l'induction moyenne dans le stator (i.e. les dents et la culasse) créée par les aimants lors d'un fonctionnement à vide, $\mathrm{L}_{\mathrm{c}}$ l'inductance cyclique et $\Phi_{\text {eff }}$ la valeur efficace du flux dans l'entrefer en fonctionnement à vide.

On définit le coefficient des pertes par courants de Foucault avec les paramètres présentés dans le tableau 3 :

$k_{f}=\frac{e_{\text {stat }}^{2} \cdot \sigma_{\text {stat }}}{24 \cdot m v_{\text {stat }}}$

et le coefficient des pertes par hystérésis :

$$
k_{h}=\frac{P_{f e r 0}-k_{f} \cdot \omega_{0}^{2} \cdot B_{\max 0}^{2}}{\omega_{0} \cdot B_{\max 0}{ }^{2}}
$$

Avec une approche classique, on peut alors calculer respectivement les pertes fer par courants de Foucault et celles par hystérésis :

$$
\begin{aligned}
& P_{f}=k_{f} \cdot \omega^{2} \cdot B_{\text {statc }}^{2} \cdot m v_{\text {stat }} \cdot V_{\text {stat }} \\
& P_{\text {hys }}=k_{h} \cdot \omega \cdot B_{\text {statc }}{ }^{2} \cdot m v_{\text {stat }} \cdot V_{\text {stat }}
\end{aligned}
$$


Nous pouvons en déduire les pertes fer totales du moteur:

$$
P_{\text {fer }}=P_{h y s}+P_{f}
$$

Les pertes fer totales du moteur sont représentées Figure 12. Nous pouvons constater que la valeur maximale des pertes fer du moteur se situe au point nominal. En régime défluxé, au delà de la vitesse nominale, l'induction diminue en même temps que la fréquence augmente, les vitesses de variation de ces deux paramètres conduisent finalement à une diminution des pertes fer.

Tableau 3. Paramètres des calculs de pertes fer de la machine

\begin{tabular}{ccc}
\hline Paramètre & Symbole & Valeur \\
Epaisseur d'une tôle statorique & $e_{\text {stat }}$ & $0.35 \mathrm{~mm}$ \\
Conductivité de la tôle statorique & $\sigma_{\text {stat }}$ & $2325581 \Omega^{-1} \mathrm{~m}^{-1}$ \\
Masse volumique du stator & $m v_{\text {stat }}$ & $7650 \mathrm{~kg} / \mathrm{m}^{3}$ \\
Pertes fer spécifique à 1.5 T et à 50 Hz & $P_{\text {fer } 0}$ & $2.7 \mathrm{~W} / \mathrm{kg}$ \\
Pulsation à 50 Hz pour les pertes fer à 1.5T & $\omega_{0}$ & $314 \mathrm{~Hz}$ \\
Induction maximale à 50 Hz & $B_{\max 0}$ & $1.5 \mathrm{~T}$ \\
\hline
\end{tabular}

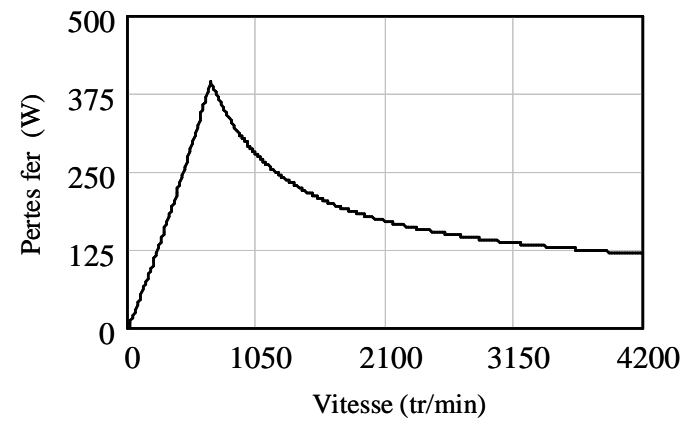

Figure 12. Pertes fer totales du moteur 


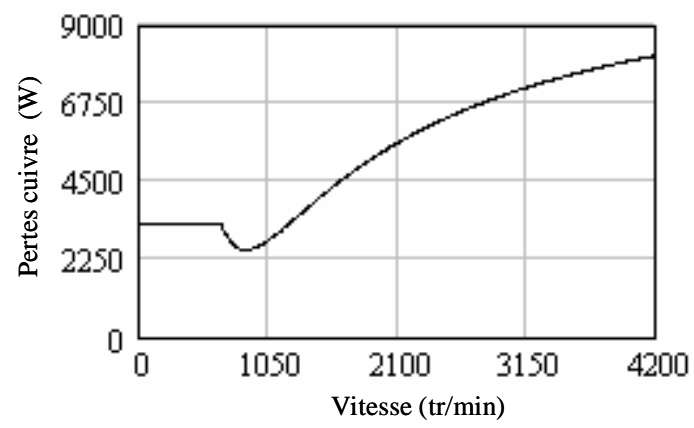

Figure 13. Pertes cuivre des bobinages statoriques du moteur

(a)

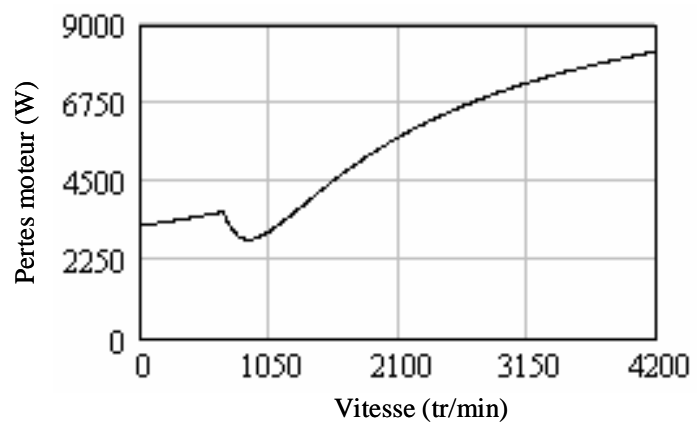

(b)

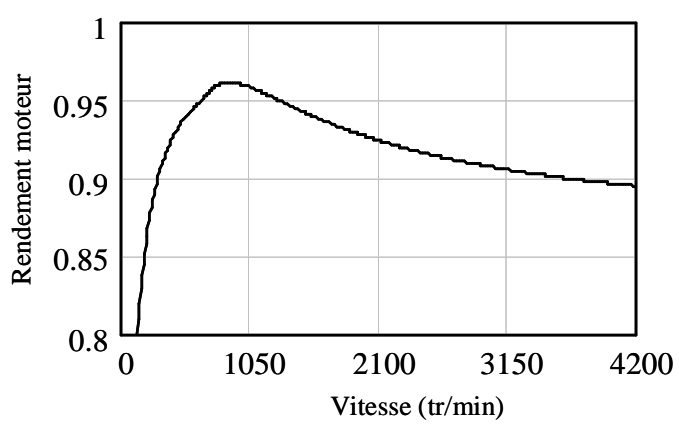

Figure 14. (a)Pertes et (b) rendement du moteur

Les pertes cuivre dans les bobinages statoriques s'expriment de la façon suivante :

$P_{c u}=n_{p h} \cdot R_{s} \cdot I_{\text {eff }}^{2}$ 
La Figure 13 donne les pertes cuivre en fonction de la vitesse. Elles sont directement liées à l'évolution du courant statorique donnée Figure 11.b. Nous pouvons calculer ensuite les pertes totales dans le moteur et le rendement du moteur, qui sont représentés Figure 14.

\section{Modélisation des pertes dans l'onduleur}

Les pertes dans l'onduleur comprennent les pertes par conduction et celles par commutation dans les transistors IGBT et les diodes de l'onduleur.

Les pertes dans l'onduleur sont exprimées analytiquement en fonction des grandeurs électriques, en supposant que le régime de fonctionnement est sinusoïdal. Le modèle analytique électromécanique décrit dans le paragraphe précédent détermine les courants circulant dans l'onduleur qui sont ensuite utilisés par le modèle de pertes. Ce dernier permet l'étude de l'évolution des pertes dans l'onduleur en fonction des lois de commande choisies (couple, puissance, vitesse, tension de bus, fréquence de commutation des semi-conducteurs de l'onduleur). Les paramètres constructeurs des IGBT et des diodes de l'onduleur, la tension d'alimentation continue et les courants dans la machine constituent les données d'entrée du modèle de pertes. Le tableau 4 liste ces caractéristiques dans le cas de modules IGBT SKM600GB066D de Semikron. Les courants sont donnés par le modèle électromécanique et évoluent avec la vitesse de rotation du moteur.

Les pertes par conduction dans chaque IGBT sont exprimées par intégration sur une période électrique $\mathrm{T}$ de la puissance instantanée aux bornes du composant comme l'exprime l'équation [12] :

$P_{\text {condT }}=\frac{1}{T} \cdot \int_{0}^{T} V_{C E s a t} \cdot I_{T}(t) \cdot d t$

Tableau 4. Caracteristiques de l'onduleur

\begin{tabular}{ccc}
\hline Paramètre & Symbole & Valeur \\
\hline Tension constructeur de mesure de $\mathrm{E}_{\text {on }}, \mathrm{E}_{\text {off }}$ & $V_{\text {Eon_off }}$ & $300 \mathrm{~V}$ \\
Courant constructeur de mesure de $\mathrm{E}_{\text {on }}, \mathrm{E}_{\text {off }}$ & $I_{\text {Eon_off }}$ & $600 \mathrm{~A}$ \\
Energie dissipée par amorçage IGBT & $E_{\text {on }}$ & $7.5 \cdot 10^{-3} \mathrm{~J}$ \\
Energie dissipée par extinction IGBT & $E_{\text {off }}$ & $29.5 \cdot 10^{-3} \mathrm{~J}$ \\
Chute de tension directe IGBTs & $V_{C E s a t}$ & $1.9 \mathrm{~V}$ \\
Fréquence de commutation & $F_{\text {com }}$ & $10000 \mathrm{~Hz}$ \\
Chute de tension directe Diodes & $V_{F 0}$ & $1 \mathrm{~V}$ \\
Energie dissipée par extinction Diodes & $E_{r r}$ & $25 \cdot 10^{-3} \mathrm{~J}$ \\
Tension continue d'entrée & $\mathrm{V}_{\text {bus }}$ & $540 \mathrm{~V}$ \\
\hline
\end{tabular}


En partant de l'hypothèse que la modulation est de type sinusoïdale intersective, le rapport cyclique s'exprime par l'équation [13] :

$\alpha(t)=\frac{1}{2}+m \cdot \sin (\omega t+\varphi)$

où $\mathrm{m}$ représente le taux de modulation et $\varphi$ le déphasage entre la tension simple $\mathrm{v}(\mathrm{t})$ et le courant $\mathrm{i}(\mathrm{t})$ d'une même phase, ce dernier étant pris comme référence. On peut alors en déduire le courant dans les IGBTs en fonction du courant de phase et ensuite les pertes par conduction dans les IGBT. Elles sont données par l'expression [14] cidessous où $I_{\max }$ et $V_{\max }$ représentent respectivement les amplitudes du courant de phase et de la tension simple :

$$
P_{\text {condT }}=\frac{V_{\text {CEsat }} \cdot I_{\max }}{2} \cdot\left[\frac{1}{\pi}+\frac{V_{\max }}{2 \cdot V_{\text {bus }}} \cdot \cos (\varphi)\right]
$$

Les pertes par commutation dans les IGBT sont obtenues en extrapolant les valeurs d'énergie dissipée données par le constructeur par une approximation linéaire en fonction des tensions et courants observés lors des commutations, approximation justifiée pour le type de semi-conducteurs utilisés. Ainsi la puissance dissipée par amorçage et par transistor est définie comme la somme des énergies dissipées à l'amorçage pendant une seconde comme l'exprime l'équation [15]:

$$
P_{\text {onT }}=\sum_{i=1}^{N(1 s)} E_{\text {on }} \frac{I(i)}{I_{\text {Eon_off }}} \cdot \frac{V_{\text {bus }}}{V_{\text {Eon_off }}}
$$

En approximant la répartition des commutations comme étant uniforme sur l'alternance positive, la valeur moyenne du courant sur l'alternance considérée peut se substituer au courant lors des commutations. Les pertes à l'amorçage par IGBT s'expriment donc par l'équation [16] :

$$
P_{\text {onT }}=\frac{E_{\text {on }} \cdot I_{\max } \cdot V_{\text {bus }}}{\pi \cdot I_{\text {Eon_off }} \cdot V_{\text {Eon_off }}} \cdot F_{\text {com }}
$$

En procédant de la même manière pour les pertes à l'extinction des IGBTs, on obtient l'équation [17] :

$$
P_{\text {off } T}=\frac{E_{\text {off }} \cdot I_{\max } \cdot V_{\text {bus }}}{\pi \cdot I_{\text {Eon_off }} \cdot V_{\text {Eon_off }}} \cdot F_{\text {com }}
$$


Les pertes totales par IGBT s'expriment alors simplement par l'équation [18] cidessous :

$$
P_{T}=P_{c o n d T}+P_{o n T}+P_{o f f T}
$$

Les pertes dans les diodes sont obtenues en appliquant les mêmes méthodes de calcul que pour les transistors et en tenant compte d'une conduction complémentaire à celle des IGBT sur chaque intervalle de période porteuse. Ainsi les équations [19] et [20] permettent d'exprimer respectivement les pertes par conduction et par commutation dans chaque diode de l'onduleur.

$$
\begin{aligned}
& P_{\text {condD } D}=\frac{V_{F O} \cdot I_{\max }}{2} \cdot\left[\frac{1}{\pi}-\frac{V_{1 \max }}{2 \cdot V_{\text {bus }}} \cdot \cos (\varphi)\right] \\
& P_{\text {comD }}=\frac{E_{r r} \cdot I_{\max } \cdot V_{\text {bus }}}{\pi \cdot I_{\text {Eon_off }} \cdot V_{\text {Eon_off }}} \cdot F_{\text {com }}
\end{aligned}
$$

Les pertes totales par diode s'expriment alors par l'équation [21] ci-dessous :

$P_{D}=P_{\text {cond }}+P_{\text {com } D}$

Finalement, on en déduit les pertes totales dans les semi-conducteurs de l'onduleur à partir des équations [21] et [18] :

$$
P_{\text {ond }}=6 \cdot\left(P_{D}+P_{T}\right)
$$

Le modèle ainsi constitué permet de décliner rapidement le comportement dissipatif de l'onduleur en fonction du domaine de fonctionnement. La courbe de la Figure 15. (a) donne l'évolution des pertes dans l'onduleur en fonction de la vitesse du moteur dans les conditions considérées précédemment (cf. Figure 9). Le rendement peut également être représenté en fonction de la vitesse comme l'illustre la courbe de la Figure 15. (b). L'influence des paramètres sur le rendement est rapidement quantifiable et les gammes de vitesse à rendement optimal sont facilement identifiables. 
(a)

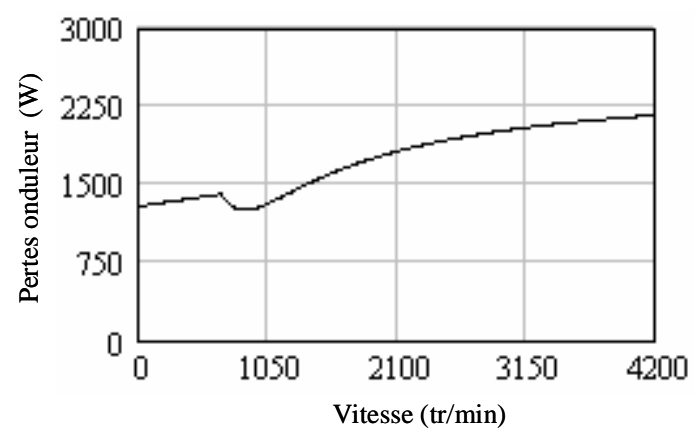

(b)

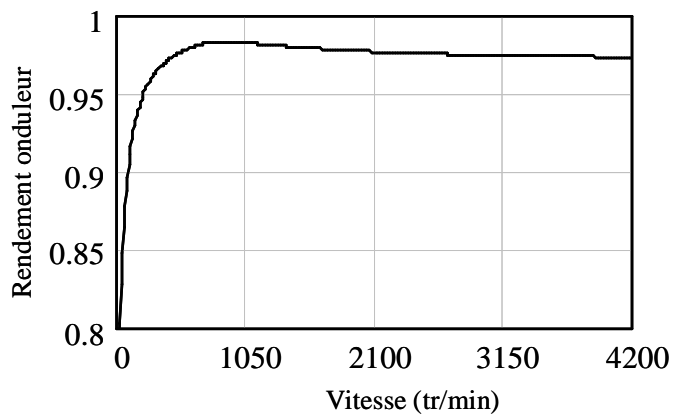

Figure 15. (a) Pertes dans les semi-conducteurs de l'onduleur (b) Rendement de l'onduleur

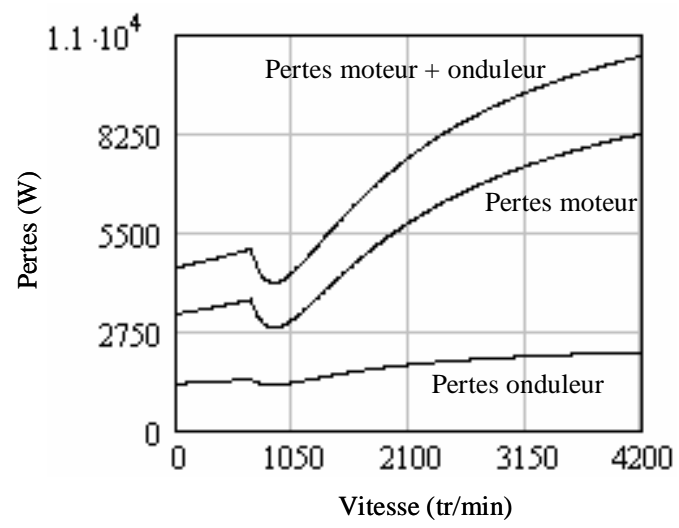

Figure 16. Pertes globales du moteur et de l'onduleur 


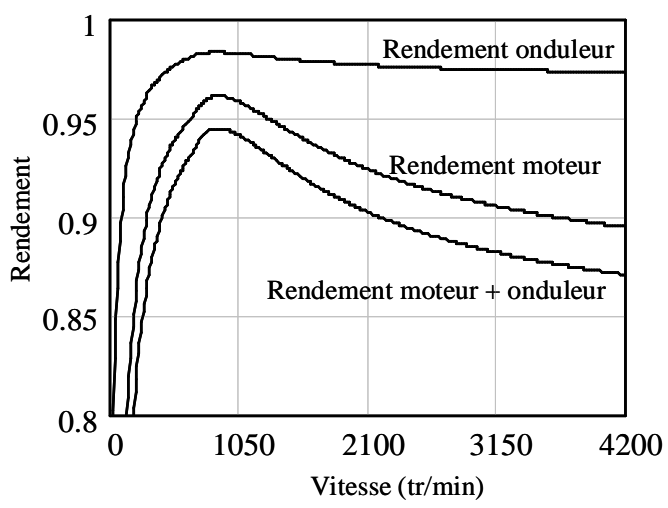

Figure 17. Rendement global de l'ensemble moteur+onduleur

Les pertes dans l'onduleur et la machine, obtenues pour les mêmes points de fonctionnement sont agrégées pour permettre une étude du rendement global de l'ensemble de la chaîne de traction. Les évolutions des pertes dans le moteur, dans l'onduleur et des pertes totales de l'entrainement sont représentées Figure 16. Cette étude est une étape importante avant la conception de la machine et du convertisseur à partir d'une optimisation énergétique globale.

Les rendements du moteur, de l'onduleur et total sont représentés Figure 17. Nous pouvons observer que les plages de fonctionnement énergétiquement optimales se situent au-delà de la vitesse de base (vitesse de fin du fonctionnement à couple maximal), ce qui correspond à la plage de fonctionnement statistiquement la plus fréquemment exploitée.

\section{Conclusion}

Dans cet article, une méthode de modélisation d'un entraînement électrique à aimants permanents utilisé dans une chaîne de traction de véhicule hybride électrique de type poids lourd est proposée. La conception de la machine synchrone à aimants permanents est basée sur la détermination des caractéristiques géométriques à l'aide d'une modélisation analytique simplifiée. La modélisation analytique et le résultat obtenu avec celle-ci sont validés par une modélisation par éléments finis. Les grandeurs électriques sont ensuite obtenues par un modèle analytique électromécanique permettant de prendre en compte les contraintes liées à l'alimentation telles que la tension du bus continu, dans la gamme de couple, puissance et vitesse considérée. Deux autres modèles analytiques permettent de calculer les pertes dans le moteur et dans l'onduleur de tension. L'utilisation séquentielle de ces modèles permet d'évaluer les propriétés et performances 
énergétiques de l'ensemble onduleur moteur vis-à-vis des différents degrés de liberté. Les pertes totales et le rendement global sont ensuite évalués afin d'optimiser la motorisation électrique. La résolution entièrement analytique et paramétrée du calcul des pertes dans la propulsion électrique a pour principal intérêt de permettre de réaliser objectivement son dimensionnement sans être tributaire d'outils de simulation dont les temps de calcul sont prohibitifs. La construction d'un modèle global à partir des modèles présentés dans cet article permettra la mise en œuvre de stratégies d'optimisation par minimisation de critères de performance, tels que les pertes totales, la masse, l'encombrement, etc. Cependant, l'obtention d'une motorisation électrique optimale ne conduit pas forcément à une chaîne de traction optimale. L'interaction entre les éléments constitutifs de la chaîne de traction (éléments de stockage, transmission mécaniques, moteurs électriques et thermique, convertisseurs électroniques) doit être prise en compte dès la conception. Cet article constitue en cela l'étape initiale d'une modélisation conjointe des éléments en vue d'une optimisation globale de la chaîne de traction.

Remerciements : Ce travail a été réalisé en collaboration avec Nexter Systems dans le cadre du projet ARCHYBALD de l'édition 2007 du programme ANR/PREDIT Véhicules Propres et Economes. Il est financé par l'Agence Nationale de la Recherche (ANR) et soutenu par l'Agence de l'Environnement et de la Maîtrise de l'Energie (ADEME).

\section{Bibliographie}

Brisset S., Démarches et Outils pour la Conception Optimale des Machines Electriques, Habilitation à Diriger des Recherches, Université des sciences et technologies de Lille, Décembre 2007.

Capitaneanu S.L, Optimisation de la fonction MLI d'un onduleur de tension deux-niveaux, Thèse de doctorat, Institut National Polytechnique de Toulouse, Novembre 2002.

Cecco E., Contribution au développement d'un outil logiciel d'aide à la conception de systèmes électromagnétiques. Application aux machines synchrones à aimants permanents, Thèse de doctorat, Université Paris Sud XI, Juillet 2005.

Chan C.C., The State of the Art of Electric and Hybrid Vehicles », Proceedings of the IEEE, Vol. 90, No. 2, February 2002.

Chan CC., Electric, Hybrid, and Fuel-Cell Vehicles: Architectures and Modeling, IEEE Transactions on Vehicular Technology, Vol. 59, No. 2, February 2010, pp. 589-596

Depernet D., Optimisation de la commande d'un onduleur MLI à trois niveaux de tension pour machine asynchrone, Thèse de doctorat, Université de Reims Champagne-Ardenne, Décembre 1995.

Espanet C., Modélisation et conception optimale de moteurs sans balais à structure inversée. Application au moteur-roue, Thèse de doctorat, Université de Franche-Comté, Janvier 1999. 
Gasc L., Conception d'un actionneur à aimants permanents à faibles ondulations de couple pour assistance de direction automobile Approches par la structure et par la commande, Thèse de doctorat, Institut National Polytechnique de Toulouse, Novembre 2004.

Gautreau T., Estimation des pertes fer dans les machines électriques. Modèle d'hystérésis loss surface et application aux machines synchrones à aimants, Thèse de doctorat, Institut National Polytechnique de Grenoble, Décembre 2005.

Lechevallier L., Le Breton J.M., Tenaud P., Morel A., Brassard S., Aimants permanents Applications et perspectives, Techniques de l'ingénieur, RefD2102, Mai 2007.

Mester V., Conception Optimale Systémique des Composants des chaînes de Traction Electrique, Thèse de doctorat, Ecole Centrale de Lille, Mai 2007.

Regnier J., Conception de systèmes hétérogènes en Génie Electrique par optimisation évolutionnaire multicritère, Thèse de doctorat, Institut National Polytechnique de Toulouse, 2003.

Slemon G., On the Design of High-Performance Surface-Mounted PM Motors, IEEE Transactions on Industry Applications, Vol.30, No.1, pp.134 -140, January/February.1994.

Slemon G., Liu X., Modeling and design optimization of permanent magnet motors, Electrical Machines and Power Systems, Vol. 20, pp.71-92, 1992.

Taylor S.P., Design and Simulation of High Performance Hybrid Electric Vehicle Powertrains, Thesis of Master, College of Engineering and Mineral Resources at West Virginia University, 2001.

Zhu L., Jiang S.Z., Zhu Z.Q. and Chan C.C., Comparison of Alternate Analytical Models for Cogging Torque in Surface-Mounted Permanent Magnet Machines, IEEE Vehicle Power and Propulsion Conference, September 2008. 